\title{
A Soft Sensing Method for Temperature of Large-Scale Aging Furnace Forgings Based on Grey System Theory
}

\author{
Dongyang Chen, Ling Shen, Jianjun He* \\ School of Information Science and Engineering, Central South University, Changsha, China. \\ *Corresponding author. Email: jjhe@csu.edu.cn \\ Manuscript submitted September 3, 2017; accepted November 28, 2017. \\ doi: 10.17706/jcp.13.7.784-793
}

\begin{abstract}
Setting proper holding time is very important to ensure the product quality during the production of large-scale aluminum forgings in the aging furnaces. The obtaining of the actual forging temperature is a prerequisite for setting the proper holding time. Due to the special furnace structure and limit of the aging technique, the forging temperature cannot be measured directly. According to the above problem, a traditional grey model GM $(1, N)$ was proposed where the symbol GM $(1, N)$ stands for first-order grey model with $\mathrm{N}$ variables, forecasting the temperature of the forgings, getting good prediction effect. But the control precision remains to be improved. Finally, an improved GM $(1, N)$ model was established by combining the equal dimension and new information model with residual modification on the basis of the experimental data. The simulation results show that the improved model has higher prediction precision.
\end{abstract}

Keywords: Aging furnace, soft measurement, grey prediction, neural network.

\section{Introduction}

The large-scale extrusion aging furnace is the key equipment for aging treatment of the military industry aluminum products. These aluminum alloy products after aging treatment are important parts of the aviation spacecraft, such as aircraft, missiles, and rockets. To obtain high strength mechanical properties and uniform crystal structure, the temperature range of the aging heat treatment is very narrow, generally achieving $-3{ }^{\circ} \mathrm{C} \sim 3{ }^{\circ} \mathrm{C}$ and the control accuracy of about $-1{ }^{\circ} \mathrm{C} \sim 1{ }^{\circ} \mathrm{C}$. The large-scale extrusion aging furnace with the length of $28 \mathrm{~m}$ has complex working condition and adopts multi-section heating method. Furthermore, the distribution of the furnace temperature is intrinsic non-uniformity. In the actual production process, the furnace is an enclosed reactor and the untreated forgings is placed in the middle of the aging furnace, leading to the great difficulty in measuring the forgings' temperature directly. For the aging treatment of high-quality forgings, it can't reach the required control precision if the temperature data of the furnace wall are used as the feedback information in the closed-loop control of the heating process. Therefore, it inevitably results in unqualified products of inadequate aging or overaging.

To solve the above problem, some researchers have concentrated on the development of soft sensing methods during the last few decades. These methods can be divided into mechanism model [1] and data model which includes least square fitting model [2], time series model [3], artificial neural network [4], support vector machine [5], and system identification [6], etc. However, it is impossible to have a comprehensive understanding for the large-scale aging furnace because of its complex working conditions. Considering the difficulty in measuring temperature directly, obtaining the sampling data is also a little bit 
difficult. An indirect method for measuring the forging temperature is put forward in this paper, namely the temperature soft sensing method based on grey prediction model. First of all, the suitable auxiliary variables are found out through analyzing the structure and production process of the aging furnace. Then, the temperature of the forgings can be predicted by establishing the traditional grey prediction model based on the grey system theory. The prediction effect is good, although there are still shortcomings. Experiments show that new data is more meaningful to establish an accurate prediction model compared with the old data through the analysis of the input and output data. So the equal dimension and new information model of grey prediction was established. At the same time, the second optimization was achieved based on the residual error which is the difference between the prediction value and the actual value. Simulation results show that the optimized prediction model can reach the required prediction accuracy and satisfy the production requirements of high quality and high precision.

The remaining parts of this paper are organized as follows. Section 2 briefly introduces the structure of the large-scale aging furnace and the process of the thermal treatment of aluminum alloy forgings. In Section 3, Grey system theory is introduced, firstly. Then, two improved algorithms are applied to modify the original model. In Section 4, the simulation results are presented here. Section 5 summarizes the conclusion.

\section{The Structure and Production Process of the Aging Furnace}

\subsection{The Structure of the Aging Furnace}

As illustrated in Fig. 1, the aging furnace with the length of $28 \mathrm{~m}$ is separated into two layers by a thin insulating plate, the upper for the heating chamber, the lower for the working chamber. The original aluminum alloy forgings are placed in the centre of the aging furnace by a skip car through the guide rail. In order to ensure the quality of ventilation and enhance the temperature distribution uniformity of the furnace, the mineral wool slag is used as the thermal insulation material of the furnace and the stainless steel is used as the material of interlayer sheet. The heating mode of the aging furnace is centralized heating mode. In the heating process, the heat generated by the heating elements which are installed at the top of the aging furnace, is flew to the working chamber by two sets of high-power fans through air deflectors from two directions regularly. And the fans are installed at the middle and upper parts of the furnace. The concentrated heating mode is different from the single district heating mode because the temperature distribution uniformity is better. Temperature of the furnace is regulated by conduction and disconnect of the heating elements' electrical current. Thermocouples are distributed on the wall of the working chamber.

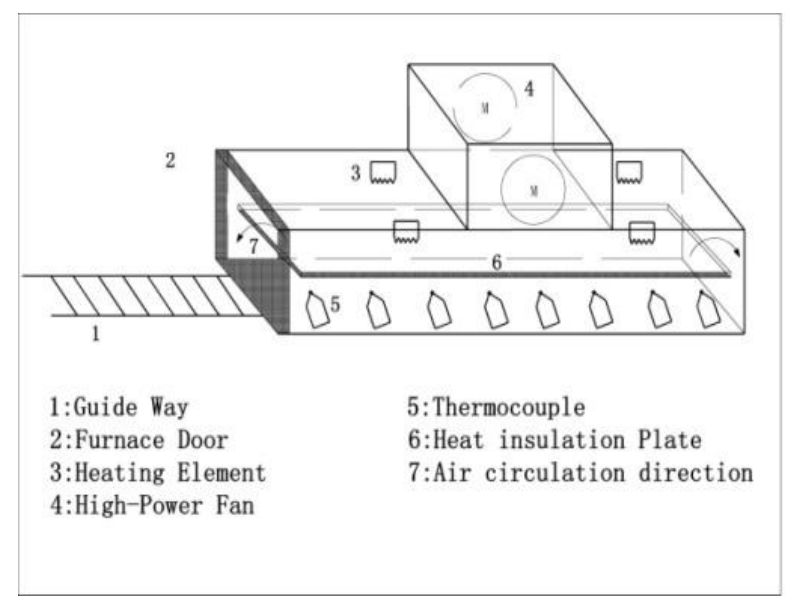

Fig. 1. Structural drawing of the furnace. 


\subsection{The Production Process}

Aging process is the most important heat treatment process for producing large-scale high strength aluminum alloy forgings. Aluminum alloy treated by aging process will not only have a great improvement on mechanical strength, but also eliminate the residual stress inside the forgings, ensure the machining accuracy, prevent crack, and improve the overall quality of forgings. Producing high-quality aluminum alloy not only depends on the kind of additional metal elements, impurity elements and trace elements, but also relates to appropriate heat treatment process closely. Aging treatment mainly includes two phases: heating up and holding period. As illustrated in Fig. 2, the temperature of the furnace wall is always lower than the actual forging temperature. When there is no direct measurement of forgings' temperature, the time of forgings reaching the setting temperature can't be known. If the real forging temperature cannot be obtained, the time of the forgings reaching the setting temperature can't be known, and the appropriate holding time can not be set. As a result, there will be unqualified aluminum alloy products. So the actual temperature of forgings which can be acquired in real time plays a vital role to guarantee the quality of forgings.

\section{Grey System Theory}

\subsection{Grey Prediction}

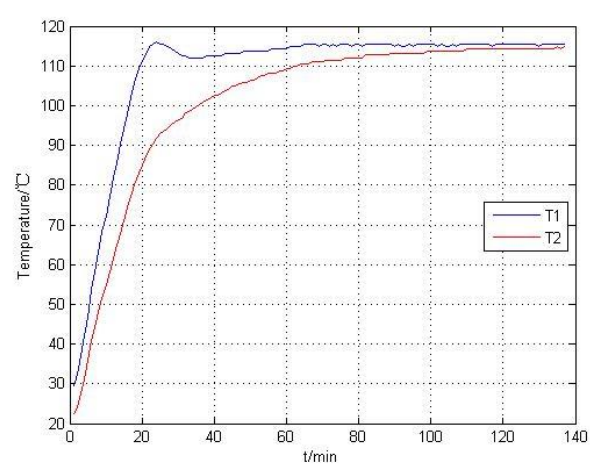

Fig. 2. Temperature variation profiles of aging furnace during the whole stage of aging treatment. The curve

$\mathrm{T} 1$ denotes the temperature of the furnace wall and $\mathrm{T} 2$ indicates the temperature of the forgings.

The grey system theory [7] was put forward by professor Deng in 1982. During the last two decades, it has been basically set up the structure of an emerging discipline system. Grey prediction uses system behavior data as the sample information, exploring the law of system development by processing system behavior data [8]. Then, it uses the law to predict the prospective behavior data of the system. It has the characteristics of strong adaptability, small amount of calculation, using conveniently and calculating quickly. At the same time, it needs less original data to establish the model and has no need to establish the model of the controlled object. Above all, it is suitable for the complex dynamic process and can satisfy the real-time control of the system [9]. Grey forecasting model is composed of a set of grey differential equations. The symbol can be written as GM (n, $m$ ), where $n$ is the order of the difference equation and $m$ is the number of the variables. Although various types of grey models can be mentioned, GM $(1, \mathrm{~N})$ is one of the most widely used grey forecasting model, which consists of a multivariable first-order differential equation [10]. Objective system has the characteristics of complex appearance and discrete data. But it is ordered and always have the overall function. So it inevitably contains certain inherent law. Grey system theory explores the law of numbers by sorting the original data. It is called generation of numbers, which is a means of predicting numbers by numbers. Assuming that there are original output sequence: 


$$
x_{1}^{(0)}=\left\{x_{1}^{(0)}(1), x_{1}^{(0)}(2) \cdots, x_{1}^{(0)}(n)\right\}
$$

and the original input sequence:

$$
\begin{aligned}
& x_{i}^{0}=\left\{x_{i}^{(0)}(1), x_{i}^{(0)}(2) \cdots \cdots x_{i}^{(0)}(n)\right\}, \\
& i=2,3 \cdots \cdots N
\end{aligned}
$$

The accumulated generating operation (AGO) is used to convert chaotic series $x_{i}^{(0)}$ into monotonically increasing series:

$$
x_{i}^{(1)}=\left\{x_{i}^{(1)}(1), x_{i}^{(1)}(2) \cdots x_{i}^{(1)}(n)\right\}
$$

where $i=1,2 \cdots N$ and $x_{i}^{(1)}$ is derived as follows:

$$
x_{i}^{(1)}(k)=\sum_{j=1}^{k} x_{i}^{(0)}(j), k=1,2 \cdots \cdots n, i=1,2 \cdots \cdots N
$$

Let $z_{1}^{(1)}$ be the sequence generated from $x_{1}^{(1)}$ by adjacent neighbor mean, that is

$$
z_{1}^{(1)}(k)=0.5 \times\left[x_{1}^{(1)}(k)+x_{1}^{(1)}(k-1)\right], k=1,2 \cdots \cdots \cdot n
$$

Establish the GM(1,N) model by using a first-order differential equation:

$$
x_{1}^{(0)}(k)+a z_{1}^{(1)}(k)=\sum_{i=2}^{N} b_{i} x_{i}^{(1)}(k)
$$

where a and bi are referred to as the development coefficient and grey action quantities, respectively. Then a and bi constitute the coefficients vector:

$$
P_{N}=\left[a, b_{2}, \cdots b_{N}\right] .
$$

Using least mean square estimation technique, PN can be estimated as:

$$
P_{N}=\left(B^{T} B\right)^{-1} B^{T} y_{N}
$$

where

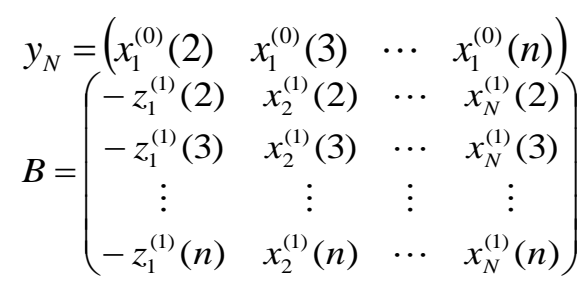

The approximate time response sequence of $\mathrm{GM}(1, \mathrm{~N})$ in Eq. 1 is given below :

$$
\begin{aligned}
& x_{1}^{(1)}(k+1)=\left[X_{1}^{(1)}(0)-\frac{1}{a} \sum_{i=2}^{N} b_{i} X_{i}^{(1)}(k+1)\right] \times \\
& \exp (-a k)+\frac{1}{a} \sum_{i=2}^{N} b_{i} X_{i}^{(1)}(k+1)
\end{aligned}
$$

where $\bar{x}_{1}^{(1)}(k)$ denotes the prediction at a given time $\mathrm{k}$, and $x_{1}^{(1)}(0)$ is taken to be $x_{1}^{(0)}(0)$.By performing 
inverse accumulated generating operation, the outputs of $\mathrm{GM}(1, \mathrm{~N})$ can be gotten:

$$
\bar{x}_{1}^{(0)}(k+1)=\bar{x}_{1}^{(1)}(k+1)-\bar{x}_{1}^{(1)}(k)
$$

\subsection{Improved Algorithm and Improvement of the Grey Prediction Model}

\subsubsection{Rolling prediction algorithm of the equal dimension and new information}

As analyzed above, the precision of the model GM $(1, N)$ is closely related to the modeling data:

$$
x_{i}^{0}=\left\{x_{i}^{(0)}(1), x_{i}^{(0)}(2) \cdots \cdots, x_{i}^{(0)}(n)\right\}
$$

In order to improve the prediction precision of the model, prevent data expansion and take disturbance and input of the system into consideration constantly, the equal dimension new information model can be established because new sequence will bring new information that old sequence doesn't include. In order to keep the number of data used to establish the model remain unchanged, new sequence is added while the same count of old sequence is removed. This is the basic idea of rolling prediction algorithm of the equal dimension and new information model [11], [12]. In GM $(1, \mathrm{~N}), x_{1}^{(0)}(k+1)$ is predicted using the data sequence:

$$
\begin{aligned}
& x_{i}^{0}=\left(x_{i}^{(0)}(k-m+1), x_{i}^{(0)}(k-m+2), \cdots, x_{i}^{(0)}(k)\right) \\
& i=1,2 \cdots N \quad m<k<n .
\end{aligned}
$$

where $\mathrm{m}$ denotes modeling dimension and $\mathrm{n}$ is taken to be the length of the data that will be predicted.

First of all, the value of $x_{1}^{(1)}(k+1)$ can be gotten through the following formula:

$$
\begin{aligned}
& \operatorname{X}_{1}^{(1)}(k+1)=\left[X_{1}^{(1)}(k-m+1)-\frac{1}{a} \sum_{i=2}^{N} b_{i} x_{i}^{(1)}(k+1)\right] \times \\
& \exp (-a m)+\frac{1}{a} \sum_{i=2}^{N} b_{i} X_{i}^{(1)}(k+1)
\end{aligned}
$$

In the same way, $x_{1}^{(1)}(k)$ is predicted using the data sequence:

$$
\begin{aligned}
& x_{i}^{0}=\left(x_{i}^{(0)}(k-m), x_{i}^{(0)}(k-m+1), \cdots, x_{i}^{(0)}(k-1)\right) \\
& i=1,2 \cdots N \quad m<k<n .
\end{aligned}
$$

through the following formula:

$$
\begin{aligned}
& \bar{X}_{1}^{(1)}(k)=\left[x_{1}^{(1)}(k-m)-\frac{1}{a^{\prime}} \sum_{i=2}^{N} b^{\prime}{ }_{i} x_{i}^{(1)}(k)\right] \times \\
& \exp \left(-a^{\prime} m\right)+\frac{1}{a^{\prime}} \sum_{i=2}^{N} b_{i}^{\prime} x_{i}^{(1)}(k)
\end{aligned}
$$

where $a^{\prime}, b_{i}^{\prime}$ are gotten by repeating the formulas(1) and(2).

Finally, the value of $x_{1}^{(0)}(k+1)$ can be gotten through the following formula:

$$
\bar{x}_{1}^{(0)}(k+1)=\bar{x}_{1}^{(1)}(k+1)-\bar{x}_{1}^{(1)}(k)
$$

After the result is found, the calculating procedure is repeated, but this time the newly got value $x_{1}^{(0)}(k+1)$ is added at the end of the data sequence, and the oldest data $x_{1}^{(0)}(k-m+1)$ is removed. Then 
the data sequence:

$$
\begin{aligned}
& x_{i}^{0}=\left(x_{i}^{(0)}(k-m+2), x_{i}^{(0)}(k-m+3), \cdots, x_{i}^{(0)}(k+1)\right) \\
& i=1,2 \cdots N \quad m<k<n .
\end{aligned}
$$

is employed to predict the value of $x_{1}^{(0)}(k+2)$. When $k=4,5, \ldots, n-1$, the whole prediction procedure is finished .This calculating method is called equal dimension rolling training algorithm, which takes into account the amount of calculation and gradually decreases the influence of the oldest data.

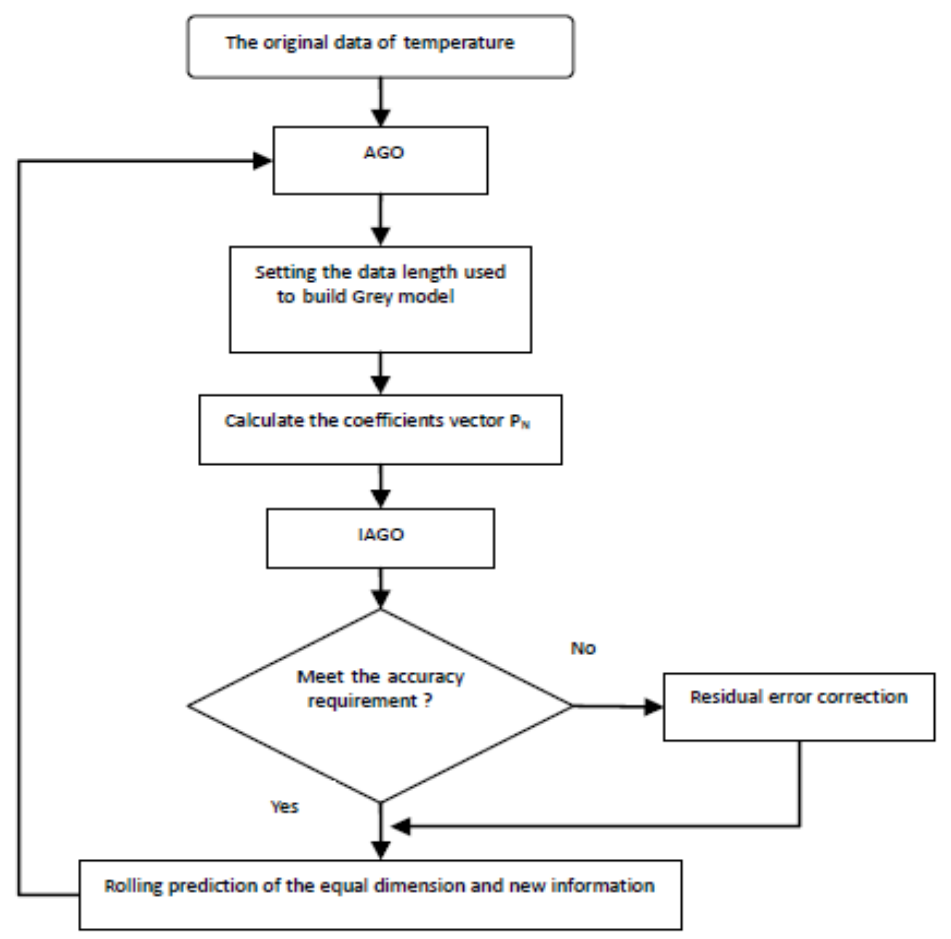

Fig. 3. Algorithm flowchart of improved grey prediction model.

\subsubsection{Residual error correction model}

Residual is the difference between sampling values and predicted values of the model. In general, the grey model should be checked out before being used. If the prediction precision of the model is not satisfied, the residual error sequence can be used to establish THE GM $(1,1)$ model to modify the original model [13].The residual error sequence can be expressed as:

$$
\varepsilon^{(0)}(k)=x^{(1)}(k)-\hat{x}^{(1)}(k)
$$

The GM $(1,1)$ model can be established according to the grey system theory:

$$
\frac{d \varepsilon^{(1)}(k)}{d t}+a_{0} \varepsilon^{(1)}(k)=b
$$

In the same way, the predicted value of the residual error sequence can be expressed as:

$$
\hat{\varepsilon}^{(0)}(k)=\left(\varepsilon^{(0)}(1)-\frac{u}{a_{0}}\right)\left(1-e^{a_{0}}\right) e^{-a_{0}(k-1)}
$$

Finally, the modified model can be gotten in combination with (4) and (9):

$$
\hat{x}_{1}^{(0)}(k)=\hat{x}_{1}^{(1)}(k)-\hat{x}_{1}^{(1)}(k-1)+\delta(k-\tau) \hat{\varepsilon}^{(0)}(k)
$$


where $\tau$ denotes the number of the residual error sequence that are used to establish the GM $(1,1)$ model. Finally, the algorithm flowchart of improved grey prediction model is illustrated in Fig. 3.

\section{Experiments and Simulation}

The experiments were conducted on the large-scale aging furnace to measure the temperature of the furnace wall and the forgings. In order to reduce the difficulty of modeling, four digital temperature sensors of eight which are in the middle of Fig. 1 are chosen as the input of the prediction model. The output of the model is the forging temperature. In order to enhance the robustness of the proposed model, the experimental data sets should cover the normal entire aging process. The temperature was measured and recorded with a sampling interval of $2 \mathrm{~min}$, including 77 data sets covering $154 \mathrm{~min}$. All temperature status of the furnace wall and forgings during the rising stage and the holding stage were captured. Fig. 4 shows the measurement results of the temperature of the furnace wall and the forgings.

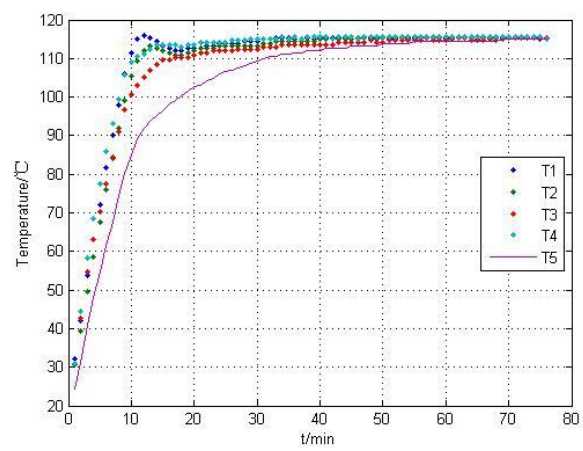

Fig. 4. Measurements of temperatures of the furnace wall and the forgings. The profiles T1, T2, T3 and T4 denote the temperature of the furnace wall separately measured. The profile $\mathrm{T} 5$ indicates the temperature of the forgings.

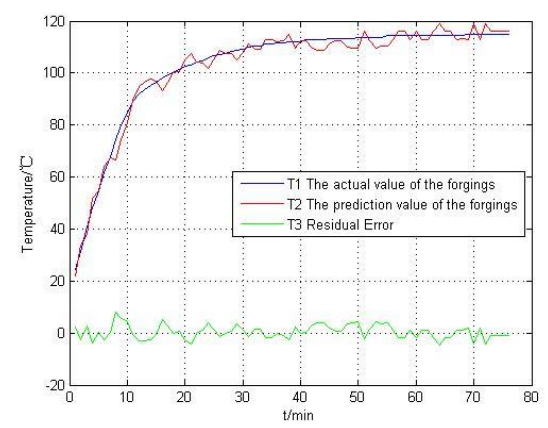

(a)

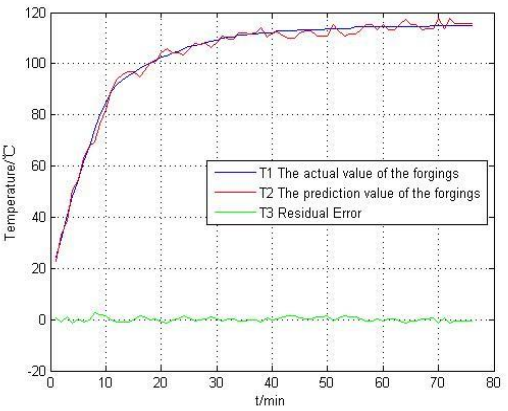

(b)

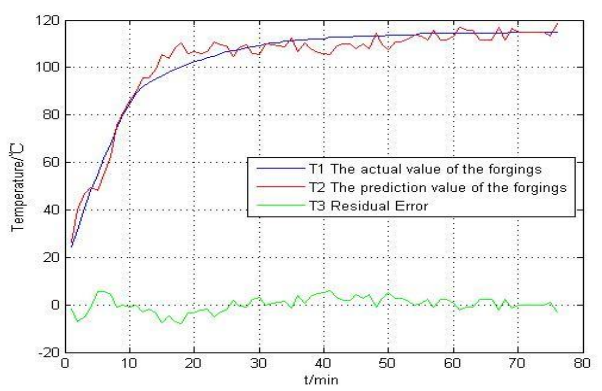

(c)

Fig. 5. Model validation results: (a): The traditional grey model (GM) (b): The improved grey model(IGM) (c):BP network. 
As shown in Fig. 4, a GM $(1,5)$ model can be established which includes 4 variables for input and 1 variable for output. For model comparison, the second group of data sets was used to evaluate the robustness of GM, IGM and BP network. The results are illustrated in Fig. 5. And the comparison results are shown in Table 1 and Fig. 6.

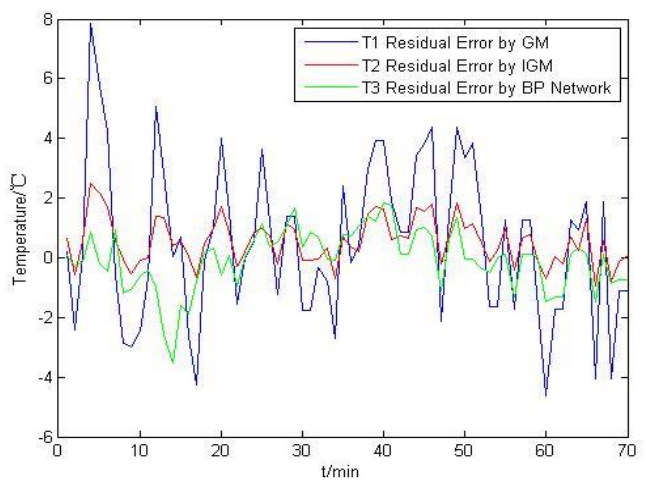

Fig. 6. Comparison of residual error.

Table 1. Comparison of the Models

\begin{tabular}{lccc}
\hline Model & Max & Mean & SD \\
\hline BP Network & 8.091 & 2.502 & 0.362 \\
GM & 7.872 & 2.130 & 0.310 \\
IGM & 3.124 & 1.213 & 0.153 \\
\hline
\end{tabular}

Some key conclusions can be deduced based on the comparison results. The forecasting profiles all reflects the overall changing trend of the temperatures. But the improved grey prediction model has the best performance on the precision of curve fitting and prediction. The precision of the improved model can satisfy the requirements of the production process. Because the improved grey model carefully takes into account of the disturbance and the driving factors brought by the new prediction data, which makes the predictive results of the improved grey prediction model has higher credibility.

\section{Conclusion}

The soft sensing technology is not only an effective method for the problem of measuring complex industrial process parameters directly, but also one of the focuses of the researches on the control field in the future. So it has extensive application prospect [14], [15]. Compared with the traditional soft sensing methods, a soft sensing method based on grey system theory has some unique advantages. It neither requires a lot of modeling and training data and nor needs a comprehensive understanding of the structure and complex internal working mechanism of the system. At the same time, it has the characteristics of a small amount of calculation and strong adaptability. So it is suitable for complex dynamic process. The working environment of the aging furnace is very complex and the uncertain factors that affect the temperature of aging furnace are numerous. All the characteristics lead it to be a typical grey system. Considering the complex working conditions of the aging furnace, the problem of measuring the forgings' temperature indirectly and less sample data sequence, the method based on the grey system theory was adopted to establish the soft sensing model of the forging temperature. What's more, the model was modified for improving the prediction precision of the model. As the simulation results show, the 
prediction model based on grey system theory can satisfy the requirements of practical production. However, The working conditions of the aging furnace are very complex. The single method can not be adapted to the various working conditions completely. We will try to build a soft measurement model of stronger adaptability and higher prediction accuracy combining with other more intelligent modeling methods.

\section{Acknowledgement}

This research was supported by National Natural Science Foundation of China (61174132) and Doctoral Fund of Ministry of Education of China (20130162110067). These financial contributions are gratefully acknowledged.

\section{References}

[1] Cheng, X., Li, S., \& Shan, S. (2007). Internal temperature field in a slab investigated based on a soft sensor model. Southern Metals, (158), 11-14.

[2] Yan, W., Zhu, H., \& Shao, H. (2003). Soft sensor modeling based on support vector machines. Journal of System Simulation, 15(10), 1494-1496.

[3] Ujjwal, K., \& Jain, V. K. (2010). Time series models (grey-markov, grey model with rolling mechanism and singular spectrumanalysis) to forecast energy consumption in India. Energy, (35), 1709-1716.

[4] Mize, C., \& Ziegert, J. C. (2000). Neural network thermal error compensation of a machining center. Precis Eng, 24(4), 338-346.

[5] Feng, R., Zhang, H., \& Shao, H. (2002). Soft seNsor modeling based on support vector machine. Information and Control, 31(6), 567-571.

[6] Yang, H., \& Ni, J. (2003). Dynamic modeling for machine tool thermal error compensation. J Manuf Sci Eng, 125(2), 245-254.

[7] Deng, J. L. (2002). The Basis of Grey Theory. Wuhan: Huazhong University of Science \& Technology Press.

[8] Cheng, Q., Cheng, Y., Wang, M., \& Wang, Y. (2011). Simulation study on model-free adaptive control based on grey prediction in ballmill load control. Chinese Journal of Scientific Instrument, 32(1), 87-92.

[9] Luo, W. (2010). Temperature fuzzy control system for frame resistance based on gray prediction model's parameter self-control. Computer Measurement\& Control, 21(4), 965-968.

[10] Yi, Z., Jianguo, Y., \& Hui, J. (2012). Machine tool thermal error modeling and prediction by grey neural network. Int J Manuf Technol, (59), 1065-1072.

[11] Ren, G., Liu, L., \& Miao, X. (2010). Prediction and implementation of the improvement grey model to electrical load. Machinery Design \& Manufacture, 2(2), 232-234.

[12] Mu, G., Li, Q., Meng, Q., \& Huan, Y. (2013). System Modeling. Beijing: National Defense Industry Press.

[13] Xiao, J., Sun, D., \& Qin, Y. (2005). Application of the grey model in electric power predicting and its optimization. Control Theory and Applicatins, 24(2), 18-21.

[14] Pan, L., Li, D., \& Ma, J. (2008). Ruan Ce Liang Ji Shu Yuan Li Yu Ying Yong. Beijing: China Electric Power Press.

[15] Yu, J. (2008). Soft sensing technology and its application. Process Automation Instrumentation, 29(1), $1-7$.

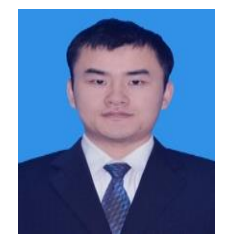

Dongyang Chen received the B.S degree in automatic control from the He Nan Polytechnic University, China in 2011. He is currently pursuing his M.S degree in Central South University. His main research interests include modeling and optimal control of distributed parameter systems, and multivariable decoupling control. 
Ling Shen received the B.S degree in automatic control from the HeNan University, China in 2008, the M.S degree in automatic control from the XiangTan University, China, in 2012. She is currently pursuing her Ph.D degree in Central South University. Her main research interests include modeling and optimal control of distributed parameter systems, and multivariable decoupling control.

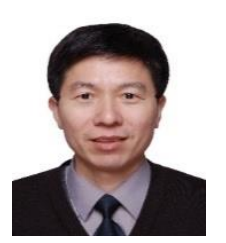

Jianjun He received the B.S degree in automatic control from the Central North University, TaiYuan City, in 1989, the M.S degree in automatic control from the Central South University, Chang Sha City, in 1992, and the Ph.D degree in mechatronics engineering from Central South University, Chang Sha City in 2004. He is currently a full professor in Central South University. His research interests include modeling, control and optimization of complicated system and production process, and adaptive control theory and application. 